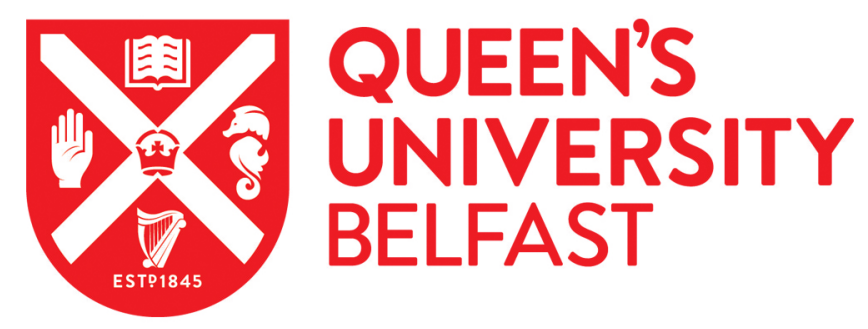

\title{
A Double-Shadowed Rician Fading Model: A Useful Characterization
}

Browning, J., Cotton, S., Morales-Jimenez, D., Sofotasios, P. C., \& Yacoub, M. D. (2020). A Double-Shadowed Rician Fading Model: A Useful Characterization. In 22nd International Symposium on Wireless Personal Multimedia Communications (WPMC): Lisbon, Portugal Nov. 24-27, 2019, (pp. 6-11). (2019 22nd International Symposium on Wireless Personal Multimedia Communications (WPMC)). Institute of Electrical and Electronics Engineers Inc.. https://doi.org/10.1109/WPMC48795.2019.9096120

\section{Published in:}

22nd International Symposium on Wireless Personal Multimedia Communications (WPMC)

\section{Document Version:}

Peer reviewed version

\section{Queen's University Belfast - Research Portal:}

Link to publication record in Queen's University Belfast Research Portal

\section{Publisher rights}

Copyright 2019 IEEE. This work is made available online in accordance with the publisher's policies. Please refer to any applicable terms of use of the publisher.

\section{General rights}

Copyright for the publications made accessible via the Queen's University Belfast Research Portal is retained by the author(s) and / or other copyright owners and it is a condition of accessing these publications that users recognise and abide by the legal requirements associated with these rights.

Take down policy

The Research Portal is Queen's institutional repository that provides access to Queen's research output. Every effort has been made to ensure that content in the Research Portal does not infringe any person's rights, or applicable UK laws. If you discover content in the Research Portal that you believe breaches copyright or violates any law, please contact openaccess@qub.ac.uk. 


\title{
A Double-Shadowed Rician Fading Model: A Useful Characterization
}

\author{
Jonathan W. Browning*, Simon L. Cotton*, David Morales-Jimenez*, Paschalis C. Sofotasios ${ }^{\dagger \ddagger}$ \\ and Michel D. Yacoub ${ }^{\S}$ \\ ${ }^{*}$ Centre for Wireless Innovation, ECIT Institute, Queens University Belfast, UK \\ e-mail: \{jbrowning01, simon.cotton, d.morales\}@qub.ac.uk \\ ${ }^{\dagger}$ Center for Cyber-Physical Systems, Department of Electrical and Computer Engineering, \\ Khalifa University, UAE \\ e-mail: paschalis.sofotasios@ku.ac.ae \\ $\ddagger$ Department of Electrical Engineering, Tampere University, Finland \\ e-mail: paschalis.sofotasios@tuni.fi \\ ${ }^{\S}$ Department of Communications, University of Campinas, Brazil \\ e-mail: michel@decom.fee.unicamp.br
}

\begin{abstract}
In this paper, we provide an alternative characterization for format 1 of the recently proposed double shadowed Rician fading model. Unlike the original definition, both the dominant component and the rms signal are impacted by Nakagami- $m$ processes. For this exposition, we derive analytical expressions for the envelope probability density function (PDF), the moments, the moment generating function, and the joint envelope-phase PDF. In addition, with the aid of the joint envelope-phase PDF, we investigate the phase properties of this interpretation of the double shadowed Rician fading model, while using the moments, we provide an analysis of the corresponding amount of fading.
\end{abstract}

\section{INTRODUCTION}

Composite fading models have proved very useful in the characterization of wireless fading channels, due to their ability to encapsulate the simultaneous impact of large-scale and small-scale fading [1]. Traditionally, the lognormal distribution has been a popular choice for modeling the effects of large-scale fading [2] and subsequently to form composite statistical models [2][4]. One of the key challenges with using the lognormal distribution is that it renders itself particularly difficult to handle analytically when deriving most of the performance measures which are fundamental to the analysis of fading channels. Because of this, the gamma distribution and the closely related inverse gamma distribution, which offer more favorable mathematical tractability and good approximations than the lognormal distribution have seen widespread adoption [5], [6]. These models have been used to develop a series of fading distributions which offer suitable flexibility for characterizing a range of propagation scenarios, e.g., Nakagami-m/gamma [7], Weibull/gamma [8], $\kappa-\mu /$ gamma [9], $\eta-\mu /$ gamma [10]-[12], $\alpha-\mu /$ gamma [12], $\alpha-\kappa-\mu /$ gamma [13], $\kappa-\mu /$ inverse gamma [6], and more recently the $\eta-\mu /$ inverse gamma [6].

A number of models which consider the dominant component to be fluctuating have also been proposed, examples include the shadowed Rician model [14] and the $\kappa-\mu$ shadowed model [15]. These models offer greater generalization, which renders them applicable to millimeter-wave applications [16]. Both forms of shadowing have been combined into a single statistical model through the recent proposal of the double shadowed Rician fading model [17]. In this model, the dominant component is assumed to be fluctuated by a Nakagami- $m$ random variable while the root mean square (rms) signal is assumed to undergo shadowing which is weighted by an inverse Nakagami- $m$ random variable.

In this contribution we present an alternative interpretation of the double shadowed Rician fading model. In our approach we assume that the dominant and rms signals are both perturbed by Nakagami- $m$ random processes (or equivalently gamma random processes if considering their power). In particular, we derive the envelope probability density function (PDF), moment generating function (MGF), joint envelope-phase PDF and moments of the distribution. Furthermore, we demonstrate that the joint envelope-phase PDF can be used to obtain the phase PDF of the shadowed Rician fading model [18], as well as examining the characteristics of the Amount of Fading (AF) in double shadowed Rician fading channels.

\section{THE PHysicAl MOdeL}

Consider a Rician fading channel which undergoes dominant component shadowing and a secondary round of composite shadowing. Physically, one example of how this may occur is when the direct path between the transmitter and receiver varies due to shadowing, at the same time, the total received power of the dominant and scattered components is shadowed by moving obstacles close to either the transmitter or receiver. Let $S=$ $R \exp (j \Theta)$ represent the complex signal envelope of the channel, where $R$ is the received signal envelope and $\Theta$ is the phase. Letting $X$ and $Y$ represent the in-phase and quadrature components respectively, it follows that, 
$S=X+j Y, R^{2}=X^{2}+Y^{2}, \Theta=\arg (X+j Y)$, $X=R \cos (\Theta)$ and $Y=R \sin (\Theta)$. Following from [17], the signal envelope, $R$, can be written as

$$
R^{2}=A^{2}\left[(I+\xi p)^{2}+(Q+\xi q)^{2}\right],
$$

where $I$ and $Q$ are mutually independent Gaussian random processes with $\mathbb{E}[I]=\mathbb{E}[Q]=0$ and $\mathbb{E}\left[I^{2}\right]=$ $\mathbb{E}\left[Q^{2}\right]=\sigma^{2}$, where $\mathbb{E}[\cdot]$ denotes statistical expectation. Also, $p$ and $q$ are the mean values of the in-phase and quadrature components, respectively. The Rician $k$ factor represents the ratio between the total power of the dominant component $d^{2}$, with $d^{2}=p^{2}+q^{2}$, and the total power of the scattered components $2 \sigma^{2}$, that is, $k=\frac{p^{2}+q^{2}}{2 \sigma^{2}}$. We also define a phase parameter, $\phi=\arg (p+j q)$. The dominant component is shaped by a Nakagami- $m$ random variable, $\xi$, with $\mathbb{E}\left[\xi^{2}\right]=1$ and shape parameter $m_{d}$ that controls the severity of the dominant component shadowing, with $m_{d} \rightarrow 0$ indicating severe shadowing of the dominant signal. A second Nakagami- $m$ random variable, $A$, with $\mathbb{E}\left[A^{2}\right]=1$ and shape parameter $m_{s}$, perturbs the rms of the entire received signal. The secondary shadowing severity is controlled by $m_{s}$, with $m_{s} \rightarrow 0$ representing severe shadowing of the rms signal.

\section{FUNDAMENTAL STATISTICS}

Following the model definition given in (1), the PDF of the received signal envelope, $R$, conditioned on the secondary shadowing $A$, is expressed as [17]

$$
\begin{aligned}
f_{R \mid A}(r \mid \alpha)= & \frac{2 r(1+k) e^{-\frac{r^{2}(1+k)}{\alpha^{2} \hat{r}^{2}}}}{\alpha^{2} \hat{r}^{2}\left(k+m_{d}\right)^{m_{d}} m_{d}^{-m_{d}}} \\
& \times{ }_{1} F_{1}\left(m_{d} ; 1 ; \frac{k(1+k) r^{2}}{\alpha^{2} \hat{r}^{2}\left(k+m_{d}\right)}\right),
\end{aligned}
$$

where $\hat{r}^{2}=\mathbb{E}\left[R^{2}\right]$ and ${ }_{1} F_{1}(\cdot ; \cdot ; \cdot)$ denotes the confluent hypergeometric function of the first kind. The corresponding envelope PDF can then be obtained by

$$
f_{R}(r)=\int_{0}^{\infty} f_{R \mid A}(r \mid \alpha) f_{A}(\alpha) d \alpha .
$$

Theorem 1. For $k, m_{d}, m_{s}, \hat{r}, r \in \mathbb{R}^{+}$, the envelope PDF of the double shadowed Rician fading model can be expressed as,

$$
\begin{aligned}
f_{R}(r)= & \sum_{i=0}^{\infty} \frac{4 r^{m_{s}+i}\left(m_{d}\right)_{i} m_{d}^{m_{d}} k^{i}\left(m_{s}(1+k)\right)^{\frac{m_{s}+1+i}{2}}}{\Gamma\left(m_{s}\right)(1)_{i} i ! \hat{r}^{m_{s}+1+i}\left(m_{d}+k\right)^{m_{d}+i}} \\
& \times K_{m_{s}-1-i}\left(\frac{2 \sqrt{m_{s}(1+k)} r}{\hat{r}}\right),
\end{aligned}
$$

where $\Gamma(\cdot)$ represents the Gamma function [19], $K_{v}(\cdot)$ denotes the modified Bessel function of the second kind [19] and $(\cdot)_{v}$ is the Pochhammer symbol [19].

Proof: The proof is provided in Appendix A.

Letting $\gamma$ represent the instantaneous signal-to-noise ratio (SNR), the PDF of the instantaneous SNR, $f_{\gamma}(\gamma)$, is obtained from (4) via a transformation of variables $\left(r=\sqrt{\gamma \hat{r}^{2} / \bar{\gamma}}\right)$, namely

$$
\begin{aligned}
f_{\gamma}(\gamma) & =\sum_{i=0}^{\infty} \frac{2 m_{d}^{m_{d}}\left(m_{d}\right)_{i} k^{i}\left(m_{s}(1+k)\right)^{\frac{m_{s}+1+i}{2}}}{\Gamma\left(m_{s}\right)(1)_{i} i ! \bar{\gamma}^{\frac{m_{s}+1+i}{2}}\left(m_{d}+k\right)^{i+m_{d}}} \\
& \times \gamma^{\frac{m_{s}-1+i}{2}} K_{m_{s}-1-i}\left(2 \sqrt{\frac{\gamma m_{s}(1+k)}{\bar{\gamma}}}\right),
\end{aligned}
$$

where $\bar{\gamma}=\mathbb{E}[\gamma]$ represents the average SNR.

Lemma 1. For $k, m_{d}, m_{s}, \bar{\gamma}, \gamma \in \mathbb{R}^{+}$the $M G F$ of the double shadowed Rician fading model, is given by

$$
\begin{aligned}
M_{\gamma}(s)= & \sum_{i=0}^{\infty} \frac{\left(m_{d}\right)_{i} m_{d}^{m_{d}}}{\left(m_{d}+k\right)^{m_{d} i !}}\left(\frac{m_{s}(1+k)}{s \bar{\gamma}}\right)^{\frac{m_{s}}{2}} \\
& \times\left(\frac{k \sqrt{m_{s}(1+k)}}{\sqrt{s \bar{\gamma}}\left(m_{d}+k\right)}\right)^{i} \exp \left(\frac{m_{s}(1+k)}{2 \bar{\gamma} s}\right) \\
& \times W_{-\frac{1}{2}\left(m_{s}+i\right), \frac{1}{2}\left(m_{s}-1-i\right)}\left(\frac{m_{s}(1+k)}{\bar{\gamma} s}\right),
\end{aligned}
$$

where $W_{u, v}(\cdot)$ represents the Whittaker Hypergeometric function [19].

Proof: The proof is provided in Appendix B.

Lemma 2. For $k, m_{d}, m_{s}, \bar{\gamma}, \gamma \in \mathbb{R}^{+}$the $n$-th order moment of the double shadowed Rician fading model is given by

$$
\begin{aligned}
\mathbb{E}\left[\gamma^{n}\right]= & \frac{m_{d}^{m_{d}} \Gamma\left(n+m_{s}\right) \Gamma(n+1)}{\left(m_{d}+k\right)^{m_{d}} \Gamma\left(m_{s}\right)}\left(\frac{\bar{\gamma}}{m_{s}(1+k)}\right)^{n} \\
& \times{ }_{2} F_{1}\left(m_{d}, n+1 ; 1 ; \frac{k}{m_{d}+k}\right) .
\end{aligned}
$$

where ${ }_{2} F_{1}(\cdot, \cdot ; \cdot ; \cdot)$ denotes the Gaussian hypergeometric function.

Proof: The proof is provided in Appendix C.

Proposition 1. For $k, m_{d}, m_{s}, \phi, \theta \in[-\pi, \pi)$ the joint envelope-phase PDF of the double shadowed Rician fading model, $f_{R, \Theta}(r, \theta)$ is given by (8), at the top of the next page, where

$$
\Delta(\theta, \phi)=\frac{k \cos ^{2}(\theta-\phi)}{\left(k+m_{d}\right)} .
$$

Proof: The proof is provided in Appendix D.

Proposition 2. For $k, m_{d} \in \mathbb{R}^{+}, \phi, \theta \in[-\pi, \pi)$ the phase PDF of the double shadowed Rician fading model, $f_{\Theta}(\theta)$ is shown to be the same as [18, eq. 8], namely

$$
\begin{aligned}
f_{\Theta}(\theta) & =\frac{m_{d}^{m_{d}}}{2 \sqrt{\pi}\left(k+m_{d}\right)^{m_{d}+1 / 2}}\left[\sqrt{\frac{k+m_{d}}{\pi}}\right. \\
& \times{ }_{2} F_{1}\left(m_{d}, 1 ; \frac{1}{2} ; \Delta(\theta, \phi)\right)+\frac{\Gamma\left(\frac{1}{2}+m_{d}\right) \sqrt{k}}{\Gamma\left(m_{d}\right)} \\
& \left.\times \cos (\theta-\phi)(1-\Delta(\theta, \phi))^{-m_{d}-1 / 2}\right]
\end{aligned}
$$




$$
\begin{aligned}
f_{R, \Theta}(r, \theta)= & \frac{2 r^{m_{s}} m_{d}^{m_{d}}\left(\sqrt{m_{s}(1+k)}\right)^{m_{s}+1}}{\pi\left(m_{d}+k\right)^{m_{d}} \Gamma\left(m_{s}\right) \hat{r}^{m_{s}+1}}\left[\sum_{i=0}^{\infty} \frac{\left(m_{d}\right)_{i}}{\left(\frac{1}{2}\right)_{i} i !}\left(\frac{r \sqrt{m_{s}(1+k)} \Delta(\theta, \phi)}{\hat{r}}\right)^{i} K_{m_{s}-1-i}\left(\frac{2 \sqrt{m_{s}(1+k)} r}{\hat{r}}\right)\right. \\
& \left.+\sum_{n=0}^{\infty} \frac{2 \Gamma\left(m_{d}+n+\frac{1}{2}\right)}{\left(\frac{3}{2}\right)_{n} n ! \Gamma\left(m_{d}\right)}\left(\frac{r \sqrt{m_{s}(1+k)} \Delta(\theta, \phi)}{\hat{r}}\right)^{n+\frac{1}{2}} K_{m_{s}-\frac{3}{2}-n}\left(\frac{2 \sqrt{m_{s}(1+k)} r}{\hat{r}}\right)\right]
\end{aligned}
$$

TABLE I: Special cases of the double shadowed Rician fading model

\begin{tabular}{l|c}
\hline & Fading parameters \\
\hline \hline Shadowed Rician & $m_{s} \rightarrow \infty$ \\
\hline Rician & $m_{s} \rightarrow \infty, m_{d} \rightarrow \infty$ \\
\hline Nakagami- $q$ (Hoyt) & $m_{s} \rightarrow \infty, m_{d}=0.5$, \\
& $k=\left(1-q^{2}\right) / 2 q^{2}$ \\
\hline Rayleigh & $m_{s} \rightarrow \infty, m_{d} \rightarrow \infty, k \rightarrow 0$ or \\
& $m_{s} \rightarrow \infty, m_{d}=1$ \\
\hline
\end{tabular}

Proof: The proof is provided in Appendix E.

\section{Performance Analysis}

Capitalizing on the above results, we can determine the amount of fading corresponding to the proposed composite fading model.

Corollary 1. For $k, m_{d}, m_{s} \in \mathbb{R}^{+}$the $A F$ of the double shadowed Rician fading model can be obtained as

$$
\mathrm{AF}=\frac{\left(m_{s}+1\right)\left(k^{2}+m_{d}\left(k^{2}+4 k+2\right)\right)}{m_{s}\left(1+k^{2}\right)}-1
$$

Proof: The proof is provided in Appendix F.

\section{NUMERICAL RESUlts}

The double shadowed Rician fading model comprises of a number of special cases. For instance, as $m_{s} \rightarrow \infty$, (i.e., the impact of the secondary shadowing becomes significantly reduced), the model approaches the shadowed Rician fading model. The shadowed Rician fading model also includes a number of known special cases such as Nakagami- $n$ (Rician), Rayleigh and Nakagami$q$ (Hoyt). The required parameter substitutions are summarized in Table I. Monte Carlo simulations for all these special cases are shown in Fig. 1, along with the envelope PDF of the double shadowed Rician fading model in (4).

From Fig. 2 we observe that, as $m_{s} \rightarrow 0$, the increased secondary shadowing severity causes the envelope PDF to display increasingly heavy tailed behavior. The effect is reduced as $m_{s}$ increases, with the PDF approaching that of the shadowed Rician fading model in the limit $m_{s} \rightarrow \infty$.

The joint envelope-phase PDF is of significant importance in the study of the higher order statistics of diversity systems [20] and is illustrated in Fig. 3. In the chosen example, it is evident that the most likely envelope-phase values are highly concentrated around $r=0.7, \theta=0.06$ as the probability density drops sharply as we move away from that point.

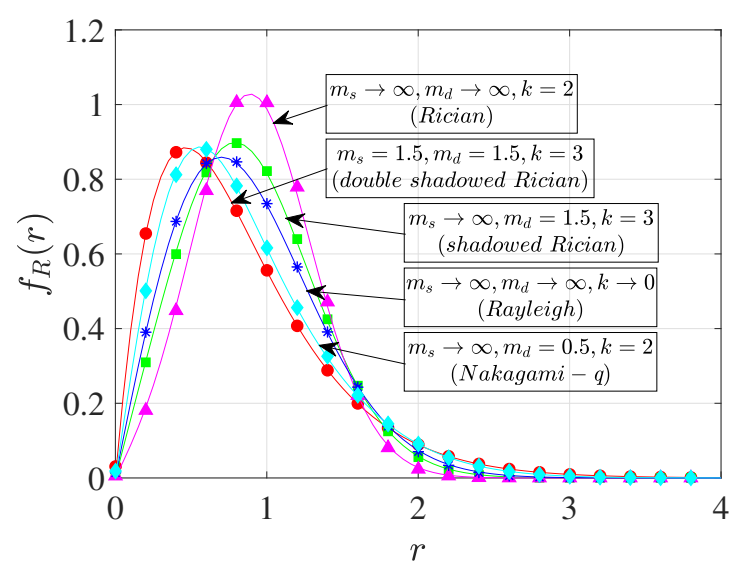

Fig. 1: The double shadowed Rician envelope PDF (4) alongside special cases. Lines represent analytical results, and circle markers represent Monte Carlo simulation results with $\hat{r}^{2}=1$.

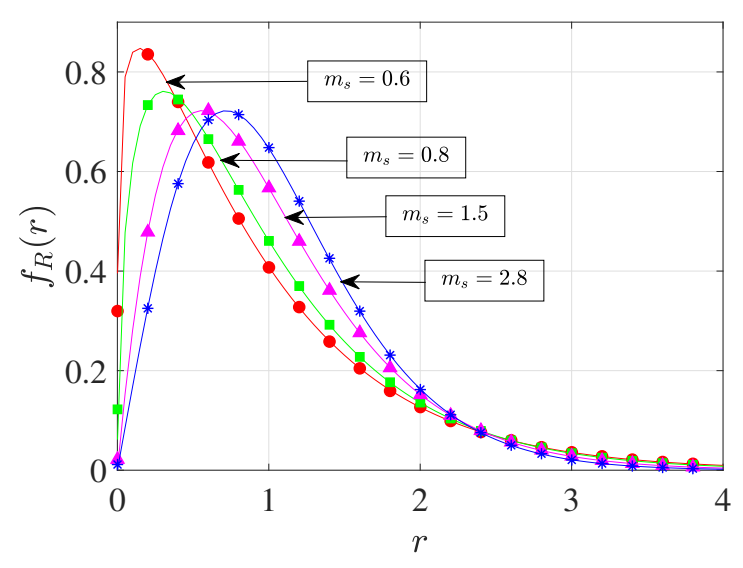

Fig. 2: The double shadowed Rician envelope PDF (4) with $m_{s}$ varying and $k=2.4, m_{d}=1.5$ and $\hat{r}^{2}=1.5$. Lines represent analytical results, and circle markers represent Monte Carlo simulation results.

The two shadowing processes (i.e., shadowing of the dominant and rms signals) have different impacts on the AF, depending on the value $k$, as illustrated in Fig. 4. For example, when $k$ is large $(e . g k=20)$, both the secondary shadowing and dominant component shadowing, respectively controlled by $m_{s}$ and $m_{d}$, affect the AF. However, when $k$ is small (e.g. $k=0.5)$, most of the shadowing is due to the rms signal fluctuations and, therefore, the AF is only significantly affected by $m_{s}$. 


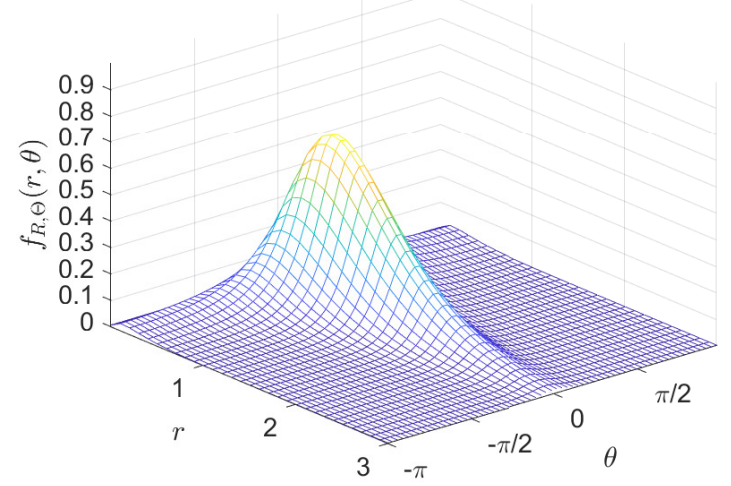

Fig. 3: The joint envelope-phase PDF (8) with $k=$ $2.4, \hat{r}^{2}=1, m_{d}=1.5, m_{s}=1.5$.

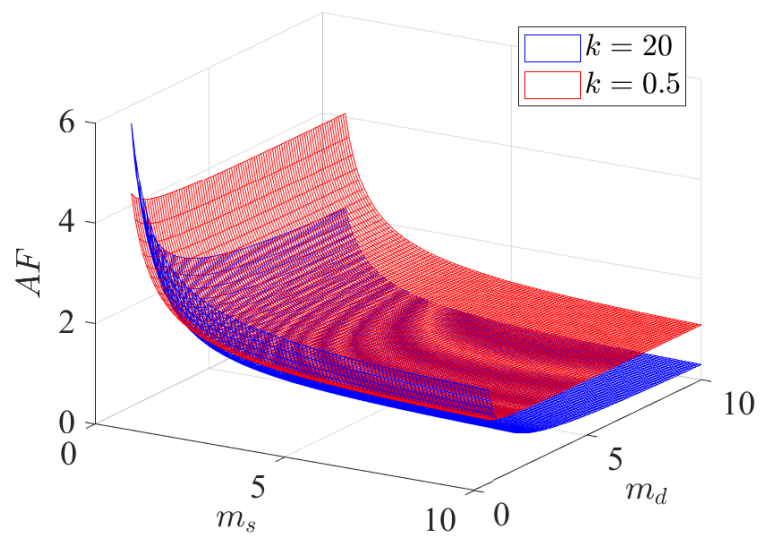

Fig. 4: The AF in double shadowed Rician channels for a range of $m_{s}$ and $m_{d}$ when $k=0.5$ and 20 .

\section{CONCLUSION}

An alternative interpretation of the double shadowed Rician fading model has been presented. In this framework, the dominant and rms signal are both subject to fluctuations caused by Nakagami- $m$ processes. Analytical solutions for the envelope PDF, MGF, moments and joint envelope-phase PDF were derived. It was also demonstrated that the joint envelope-phase PDF can be used to find the phase PDF, which is shown to be the same as in the shadowed Rician model. Using the corresponding moments, the AF of the proposed model was also found. The aforementioned model combines the advantages of a composite model and the effect of shadowing the dominant component, offering a degree of generalization that will enable it to be used in future use cases that will emerge from $5 \mathrm{G}$.

\section{APPENDIX A}

The envelope PDF is obtained by computing the integral in (3), with

$$
f_{A}(\alpha)=\frac{2 m_{s}^{m_{s}}}{\Gamma\left(m_{s}\right)} \alpha^{2 m_{s}-1} \exp \left(-m_{s} \alpha^{2}\right) .
$$

Using the series representation of the Kummer Confluent Hypergeometric function [21, eq. 07.20.06.0002.01], and a transformation of variables, (4) can be obtained from (3) by using the identity in [19, eq. 3.471.9].

\section{APPENDIX B}

The MGF can be obtained as,

$$
M_{\gamma}(s) \triangleq \int_{0}^{\infty} \exp (-s \gamma) f_{\gamma}(\gamma) d \gamma .
$$

Substituting (5) into (13) along with some mathematical manipulations, it follows that

$$
\begin{aligned}
M_{\gamma}(s)= & \sum_{i=0}^{\infty} \frac{2 m_{d}^{m_{d}}\left(m_{d}\right)_{i}\left(m_{s}(1+k)\right)^{\frac{m_{s}+1}{2}}}{\left(m_{d}+k\right)^{m_{d}} \Gamma\left(m_{s}\right)(1)_{i} i ! \bar{\gamma}^{\frac{m_{s}+1}{2}}} \\
& \times\left(\frac{k \sqrt{m_{s}(1+k)}}{\sqrt{\bar{\gamma}}\left(m_{d}+k\right)}\right)^{i} \int_{0}^{\infty} \gamma^{\frac{m s+i-1}{2}} \\
& \times \exp (-s \gamma) K_{m_{s}-1-i}\left(2 \sqrt{\frac{\gamma m_{s}(1+k)}{\bar{\gamma}}}\right),
\end{aligned}
$$

which results in (6) by using identity [19, eq. 6.643.3].

\section{APPENDIX C}

The $n$-th order moment can be calculated via, $\mathbb{E}\left[\gamma^{n}\right] \triangleq \int_{0}^{\infty} \gamma^{n} f_{\gamma}(\gamma) d \gamma$, using the PDF in (5) and the identity [19, eq. 6.561.16]; this results in

$$
\begin{aligned}
\mathbb{E}\left[\gamma^{n}\right]= & \frac{m_{d}^{m_{d}} \Gamma\left(n+m_{s}\right)}{\left(m_{d}+k\right)^{m_{d}} \Gamma\left(m_{s}\right)}\left(\frac{\bar{\gamma}}{m_{s}(1+k)}\right)^{n} \\
& \times \sum_{i=0}^{\infty} \frac{\left(m_{d}\right)_{i}}{(1)_{i} i !}\left(\frac{k}{m_{d}+k}\right)^{i} \Gamma(n+i+1) .
\end{aligned}
$$

Using the Pochhammer identity,

$$
\Gamma(x+n)=(x)_{n} \Gamma(x)
$$

and the Gauss Hypergeometric function series relationship [21, eq. 07.23.06.0002.01],

$$
{ }_{2} F_{1}(a, b ; c ; z)=\sum_{i=0}^{\infty} \frac{(a)_{i}(b)_{i} z^{i}}{(c)_{i} i !},
$$

(15) reduces to the simplified form of (7).

\section{APPENDIX D}

To find the joint envelope-phase PDF, we use a conditioning and averaging approach, similar to the one used to derive (4). Thus, all we need to do is condition the shadowed Rician joint envelope-phase PDF [18, eq. 5] with, $A$, i.e., splitting the problem into two separate integrals, namely

$$
\begin{aligned}
\mathcal{B}= & \int_{0}^{\infty} \frac{r m_{d}^{m_{d}}(1+k)}{\pi \alpha^{2} \hat{r}^{2}\left(m_{d}+k\right)^{m_{d}}} \exp \left(-\frac{r^{2}(1+k)}{\alpha^{2} \hat{r}^{2}}\right) \\
& \times{ }_{1} F_{1}\left(m_{d} ; \frac{1}{2} ; \frac{r^{2}(1+k)}{\alpha^{2} \hat{r}^{2}} \Delta(\theta, \phi)\right) f_{A}(\alpha) d \alpha,
\end{aligned}
$$


and

$$
\begin{aligned}
\mathcal{C}= & \int_{0}^{\infty} \frac{2 r^{2} m_{d}^{m_{d}}(1+k)^{3 / 2} \sqrt{\Delta(\theta, \phi)}}{\pi \alpha^{3} \hat{r}^{3}\left(m_{d}+k\right)^{m_{d} \Gamma\left(m_{d}\right)}} \\
& \times \Gamma\left(m_{d}+\frac{1}{2}\right) \exp \left(-\frac{r^{2}(1+k)}{\alpha^{2} \hat{r}^{2}}\right) \\
& \times{ }_{1} F_{1}\left(m_{d}+\frac{1}{2} ; \frac{3}{2} ; \frac{r^{2}(1+k)}{\alpha^{2} \hat{r}^{2}} \Delta(\theta, \phi)\right) f_{A}(\alpha) d \alpha .
\end{aligned}
$$

Both (18) and (19) can be found by substituting (12), doing a transformation of variables and using [19, eq. 3.471.9], yielding

$$
\begin{aligned}
\mathcal{B}= & \sum_{i=0}^{\infty} \frac{\left(m_{d}\right)_{i} 2 r^{m_{s}} m_{d}^{m_{d}}\left(r \sqrt{m_{s}(1+k)} \Delta(\theta, \phi)\right)^{i}}{\left(\frac{1}{2}\right)_{i} i ! \Gamma\left(m_{s}\right) \pi\left(m_{d}+k\right)^{m_{d}} \hat{r}^{i}} \\
& \times\left(\frac{m_{s}(1+k)}{\hat{r}^{2}}\right)^{\frac{m_{s}+1}{2}} \\
& \times K_{m_{s}-1-i}\left(\frac{2 \sqrt{m_{s}(1+k) r}}{\hat{r}}\right),
\end{aligned}
$$

and

$$
\begin{aligned}
\mathcal{C}= & \sum_{n=0}^{\infty} \frac{4 \Gamma\left(m_{d}+n+\frac{1}{2}\right) m_{d}^{m_{d}} \Delta^{n+\frac{1}{2}}(\theta, \phi)}{\left(\frac{3}{2}\right)_{n} n ! \Gamma\left(m_{d}\right) \Gamma\left(m_{s}\right) \pi \hat{r}^{\frac{1}{2}}\left(m_{d}+k\right)^{m_{d}}} \\
& \times\left(r \frac{\sqrt{m_{s}(1+k)}}{\hat{r}}\right)^{m_{s}+n+1} \\
& \times K_{m_{s}-\frac{3}{2}-n}\left(\frac{2 \sqrt{m_{s}(1+k)} r}{\hat{r}}\right) .
\end{aligned}
$$

Adding (20) and (21) together again produces the final joint envelope-phase PDF (8).

\section{APPENDIX E}

To obtain the phase PDF, we integrate the joint envelope-phase PDF (8) with respect to the envelope. This can be accomplished by using the separate components of the joint envelope-phase PDF from Appendix D i.e. (20) and (21). Using [19, eq. 6.561.16] and the series relationship of Gauss Hypergeometric function (17) results in

$$
\mathcal{B}=\frac{m_{d}^{m_{d}}}{2 \pi\left(m_{d}+k\right)^{m_{d}}} 2 F_{1}\left(m, 1 ; \frac{1}{2} ; \Delta(\theta, \phi)\right)
$$

and

$$
\begin{aligned}
\mathcal{C}= & \sum_{n=0}^{\infty} \frac{\left(m_{d}+\frac{1}{2}\right)_{n}}{\left(\frac{3}{2}\right)_{n} n !} \frac{m_{d}^{m_{d}} \sqrt{k} \Gamma\left(m_{d}+\frac{1}{2}\right)}{\pi\left(m_{d}+k\right)^{m_{d}+\frac{1}{2}} \Gamma\left(m_{d}\right)} \\
& \times \cos (\theta-\phi) \Delta(\theta, \phi)^{n} \Gamma\left(n+\frac{3}{2}\right) .
\end{aligned}
$$

A simplification of (23) can be achieved using (16) and recalling that

$$
\sum_{n=0}^{\infty} \frac{\left(m_{d}+\frac{1}{2}\right)_{n}}{n !} \Delta(\theta, \phi)^{n}=(1-\Delta(\theta, \phi))^{-m_{d}-\frac{1}{2}},
$$

$$
\mathcal{C}=\frac{m_{d}^{m_{d}} \sqrt{k} \Gamma\left(m_{d}+\frac{1}{2}\right) \cos (\theta-\phi)}{2 \sqrt{\pi}\left(m_{d}+k\right)^{m_{d}+\frac{1}{2}} \Gamma\left(m_{d}\right)(1-\Delta(\theta, \phi))^{m_{d}+\frac{1}{2}}} .
$$

When (22) and (25) are recombined we obtain the phase PDF of the shadowed Rician fading model [18, eq. 8].

\section{APPENDIX F}

The AF is defined by [2], $A F \triangleq \frac{\mathbb{E}\left[\gamma^{2}\right]}{\mathbb{E}[\gamma]^{2}}-1$. Using (7) to find $\mathbb{E}\left[\gamma^{2}\right]$, then substituting $\mathbb{E}[\gamma]^{2}$ into the $\mathrm{AF}$ equation and from [21, eq. 07.23.03.0082.01], along with some algebraic manipulations, equation (11) is deduced, which completes the proof.

\section{ACKNOWLEDGMENT}

This work was supported in part by the U.K. Engineering and Physical Sciences Research Council under Grant No. EP/L026074/1, by the Department for the Economy Northern Ireland through Grant No. USI080 and by Khalifa University under Grant No. KU/RC1-C2PS-T2/8474000137 and Grant No. KU/FSU-8474000122.

\section{REFERENCES}

[1] S. K. Yoo, S. L. Cotton, P. C. Sofotasios, and S. Freear, "Shadowed fading in indoor off-body communication channels: A statistical characterization using the $\kappa$ - $\mu$ /gamma composite fading model," IEEE Trans. Wireless Commun., vol. 15, no. 8, pp. 5231-5244, Aug. 2016.

[2] A. M.-S. Simon M. K., Digital Communications over Fading Channels, 2nd ed. New York: John Wiley, 2005.

[3] F. Hansen and F. I. Meno, "Mobile fading-Rayleigh and lognormal superimposed," IEEE Trans. Veh. Technol., vol. 26, no. 4, pp. 332-335, Nov. 1977.

[4] T. T. Tjhung and C. C. Chai, "Fade statistics in Nakagamilognormal channels," IEEE Trans. Commun., vol. 47, no. 12, pp. 1769-1772, Dec. 1999.

[5] A. Abdi and M. Kaveh, "K distribution: an appropriate substitute for Rayleigh-lognormal distribution in fading-shadowing wireless channels," IET Electron. Lett., vol. 34, no. 9, pp. 851-852, Apr. 1998.

[6] S. K. Yoo, N. Bhargav, S. L. Cotton, P. C. Sofotasios, M. Matthaiou, M. Valkama, and G. K. Karagiannidis, "The $\kappa$ - $\mu$ /inverse gamma and $\eta$ - $\mu$ /inverse gamma composite fading models: Fundamental statistics and empirical validation," IEEE Trans. Commun., pp. 1-1, 2018.

[7] I. M. Kostic, "Analytical approach to performance analysis for channel subject to shadowing and fading," IEE Proc. - Commun., vol. 152 , no. 6 , pp. $821-827$, Dec. 2005.

[8] P. S. Bithas, "Weibull-gamma composite distribution: alternative multipath/shadowing fading model," IET Electron. Lett., vol. 45, no. 14 , pp. $749-751$, Jul. 2009.

[9] P. C. Sofotasios and S. Freear, "On the $\kappa$ - $\mu /$ gamma composite distribution: A generalized multipath/shadowing fading model," in 2011 SBMO/IEEE MTT-S International Microwave and Optoelectronics Conf. (IMOC 2011), Oct. 2011, pp. 390-394.

[10] P. C. Sofotasios, and S. Freear, "The $\eta-\mu /$ gamma composite fading model,' in IEEE ICWITS '10, Honolulu, HI, USA, Aug/Sep. 2010.

[11] P. C. Sofotasios, and S. Freear, "The $\eta-\mu /$ gamma and the $\lambda-\mu /$ gamma multipath/shadowing distributions," in ATNAC '11, Melbourne, Australia, Nov. 2011.

[12] H. Al-Hmood and H. S. Al-Raweshidy, "Unified modeling of composite $\kappa$ - $\mu /$ gamma, $\eta$ - $\mu /$ gamma, and $\alpha$ - $\mu /$ gamma fading channels using a mixture gamma distribution with applications to energy detection," IEEE Antennas and Wireless Propag. Lett., vol. 16, pp. 104-108, 2017.

[13] P. C. Sofotasios and S. Freear, "The $\alpha-\kappa-\mu /$ gamma distribution: A generalized non-linear multipath/shadowing fading model," in 2011 Annual IEEE India Conf., Dec. 2011, pp. 1-6.

which leads to 
[14] A. Abdi, W. C. Lau, M. S. Alouini, and M. Kaveh, "A new simple model for land mobile satellite channels: first- and second-order statistics," IEEE Trans. Wireless Commun., vol. 2, no. 3, pp. 519-528, May 2003.

[15] J. F. Paris, "Statistical characterization of $\kappa$ - $\mu$ shadowed fading," IEEE Trans. Veh. Technol., vol. 63, no. 2, pp. 518-526, Feb. 2014.

[16] J. Kibida, Y. J. Chun, F. Firyaguna, S. K. Yoo, L. A. DaSilva, and S. L. Cotton, "Performance evaluation of millimeter-wave networks in the context of generalized fading," in 2018 IEEE Globecom Workshops (GC Wkshps), Dec. 2018, pp. 1-6.

[17] N. Bhargav, C. R. N. d. Silva, S. L. Cotton, P. C. Sofotasios, and M. D. Yacoub, "Double shadowing the Rician fading model,"
IEEE Wireless Commun. Lett., vol. 8, no. 2, pp. 344-347 Apr. 2019.

[18] J. W. Browning, S. L. Cotton, D. Morales-J., and F. J. Lopez-M., "The Rician complex envelope under line of sight shadowing," IEEE Commun. Lett., pp. 1-1 Sept. 2019.

[19] I. S. Gradshteyn and I. M. Ryzhik, Table of Integrals, Series, and Products, 7th ed. San Diego, CA, USA: Academic Press, 2007.

[20] G. Fraidenraich, J. C. S. S. Filho, and M. D. Yacoub, "Secondorder statistics of maximal-ratio and equal-gain combining in Hoyt fading," IEEE Commun. Lett., vol. 9, no. 1, pp. 19-21, Jan. 2005 .

[21] "Wolfram Research, Inc." accessed on 03/25/2019. [Online]. Available: http://functions.wolfram.com/id 